\title{
Serum $\gamma$-glutamyltransferase and the risk of heart failure in men and women in Finland
}

\author{
Yujie Wang, ${ }^{1,2,3}$ Jaakko Tuomilehto, ${ }^{4,5}$ Pekka Jousilahti, ${ }^{5}$ Veikko Salomaa, ${ }^{5}$ Bin Li, ${ }^{3}$ \\ Riitta Antikainen, ${ }^{6}$ Markku Mähönen, ${ }^{4}$ Peter T Katzmarzyk, ${ }^{1}$ Gang Hu${ }^{1}$
}

${ }^{1}$ Pennington Biomedical Research Center, Baton Rouge, Louisiana, USA

${ }^{2}$ Human Nutrition and Food Division, School of Human Ecology, Louisiana State University AgCenter, Baton Rouge, Louisiana, USA ${ }^{3}$ Department of Experimental Statistics, Louisiana State University AgCenter, Baton Rouge, Louisiana, USA ${ }^{4}$ Department of Public Health, University of Helsinki, Helsinki, Finland

${ }^{5}$ Department of Health Promotion and Chronic Diseases Prevention, National Institute for Health and Welfare, Helsinki, Finland ${ }^{6}$ Oulu University and Unit of General Practice (Geriatrics), Oulu City Hospital and Institute of Health Sciences, Oulu, Finland

\section{Correspondence to}

Dr Gang Hu, Chronic Disease Epidemiology Laboratory,

Pennington Biomedical Research Center, 6400 Perkins Road, Baton Rouge, LA 70808, USA; gang.hu@pbrc.edu

Received 31 August 2012 Revised 8 October 2012 Accepted 9 October 2012

Published Online First

9 November 2012

\footnotetext{
To cite: Wang $Y$,

Tuomilehto J, Jousilahti $P$,

et al. Heart 2013;99:

163-167.
}

\section{ABSTRACT}

Objectives To evaluate the association of serum $\gamma$-glutamyltransferase (GGT) levels with heart failure (HF) risk in the Finnish population.

Design Prospective population-based cohort study. Setting The present study, which is a part of FINRISK study, was carried out in Finland.

Subject study cohorts included 18353 Finnish men and 19726 women who were 25-74 years of age and free of $\mathrm{HF}$ at baseline.

Main outcome measures HF (636 men and 445 women) during a mean follow-up of 14.5 years.

Results Baseline measurement of different levels of serum GGT was used to predict incident HF. The multivariable-adjusted (age, sex, study area, study year, smoking, education, alcohol consumption, physical activity, valvular heart disease, body mass index (BMI), systolic blood pressure, total cholesterol at baseline, myocardial infarction and diabetes at baseline and during follow-up) HRs of HF at five GGT groups (using the 25th, 50th, 75th and 90th percentiles) were 1.00 , 1.16 ( $95 \%$ Cl: 0.97 to 1.38$), 1.20$ (1.00 to 1.45$), 1.29$ (1.04 to 1.60$)$ and 1.82 (1.45 to 2.29) $\left(P_{\text {trend }}<0.001\right)$. Stratification by smoking status, alcohol consumption and BMI gave similar results, while stronger association was observed among subjects aged $<60$ years $\left(P_{\text {trend }}=0.001\right)$ compared with subjects $60+$ years of age ( $P_{\text {trend }}=0.173$ ).

Conclusions Moderate to high levels of serum GGT (from the 50th to the 90th percentiles) were significantly associated with incident $\mathrm{HF}$ in men and women in Finland, and the predictive power was stronger in subjects aged $<60$ years.

\section{INTRODUCTION}

Heart failure (HF) is a worldwide epidemic which is associated with high morbidity and mortality. In addition to its high prevalence, HF has created heavy economic burdens on society. In the US alone, HF costs were over US\$33 billion in 2007 according to the estimation of the American Heart Association. ${ }^{1}$ In response to this severe situation, increasing attention has been drawn to identifying the risk factors of HF. Serum $\gamma$-glutamyltransferase (GGT), a widely used index of liver dysfunction, was found to be positively associated with incident cardiovascular disease (CVD), including coronary heart disease (CHD), ${ }^{2}{ }^{3}$ stroke ${ }^{3}$ and $\mathrm{HF}^{4}$ and also mortality from $\mathrm{CVD},{ }^{2}{ }^{5-7}$ including $\mathrm{CHD},{ }^{2} 56$ stroke, ${ }^{56}$ as well as congestive $\mathrm{HF}^{5-7}$ It has been proposed that the pro-oxidant effects of GGT, which result from the production of reactive oxygen species superoxide anion and hydrogen peroxide during the process of glutathione hydrolysis by GGT, might be the biological mechanism linking GGT to various cardiovascular events. ${ }^{8}$ This is supported by the findings that GGT activity has been detected in atheromatous plaques of carotid and coronary arteries where a catalytically active enzyme has been identified. ${ }^{9}$ So far, very limited information is available on the role of GGT in predicting incident HF. Thus, the aim of this study is to examine the association between GGT and the risk of incident $\mathrm{HF}$.

\section{METHODS}

\section{Participants}

Five independent cross-sectional population-based health examination surveys were carried out in six geographic areas of Finland in 1982, 1987, 1992, 1997 and 2002. ${ }^{10}$ The original random sample was stratified by area, gender and 10-year age group according to WHO Monitoring Trends and Determinants of Cardiovascular Disease protocol. ${ }^{11}$ The participation rate varied by year from $65 \%$ to $88 \%{ }^{10}$ The participants included in the five surveys were 25-64 years old, and the 1997 and 2002 surveys also included individuals 65-74 years old. Subjects who participated in more than one survey were included only in the first survey cohort. The total sample size of the five surveys was 38737 . The final sample comprised 18353 men and 19726 women after excluding the participants with a history of HF $(n=457)$ at baseline, and those with incomplete data on any variables required for this analysis $(n=201)$. The participants gave an informed consent (verbal consents in 1982-1992, and signed consents in 1997 and 2002). These surveys were conducted according to the ethical rules of the National Public Health Institute, and the investigations were conducted in accordance with the Declaration of Helsinki.

\section{Measurements}

A self-administered questionnaire was mailed to the participants to be completed at home and returned to the survey site. The questionnaire included questions on medical history, socioeconomic factors, physical activity, smoking habits and alcohol consumption. Education level, measured as the total number of school years, was divided into birth and cohort-specific tertiles. Information on occupational, commuting and leisure-time physical activity was merged and regrouped into three categories: low, moderate and high. ${ }^{12}{ }^{13}$ Participants were 
classified as never, ex-smokers and current smokers. Alcohol consumption was categorised into four groups: none, 1-34, $35-209, \geq 210 \mathrm{~g}$ per week in men; none, $1-34,35-139, \geq 140 \mathrm{~g}$ per week in women. Data on diabetes and myocardial infarction at baseline and during follow-up were obtained from the questionnaire and completed by the National Hospital Discharge Register and National Social Insurance Institution's Drug Register (diabetes only). ${ }^{14}$ Data on the history of valvular heart disease at baseline were collected by hospital discharge register. Data on liver cirrhosis at baseline and during follow-up were collected by the National Hospital Discharge Register.

At the survey site, specially trained research nurses measured participants' height and weight by using a standardised protocol. ${ }^{11}$ Body mass index (BMI) was calculated by dividing weight in kilograms by the square of height in metres. Blood pressure was measured from the right arm after $5 \mathrm{~min}$ of sitting using a mercury sphygmomanometer in each survey. After blood pressure measurement, a venous blood specimen was taken. The serum total cholesterol level was determined by an enzymatic method (CHOD-PAP, Boehringer MANNHEIM, Mannheim, Germany). GGT was determined from fresh venous blood serum samples using a kinetic method (Oy Medix Biochemica AB, Kauniainen, Finland) based on the recommendation of the European Committee for Clinical Laboratory Standards. All samples were analysed in the same central laboratory at the National Public Health Institute.

\section{Prospective follow-up}

Follow-up information was from the Finnish Hospital Discharge Register and the National Social Insurance Institution's Register on special reimbursement for HF drugs for non-fatal outcomes and the Finnish Causes of Death Register for fatal outcomes by record linkage using the personal identification numbers assigned to every citizen of Finland. ${ }^{15}$ The International Classification of Diseases (ICD) codes 427.00 and 427.10 (ICD-8), 428, 4029B (hypertensive heart disease with HF) and 4148A-X (ischemic HF with chronic CHD) (ICD-9), and I 50, I11.0 (hypertensive heart disease with HF), I13.0 and I13.2 (hypertensive heart and renal disease with HF) (ICD-10) were used to identify HF cases in any one of the above mentioned national databases. A HF diagnosis was made by the treating physicians, based on a clinical assessment and examinations as considered relevant by the clinician in charge of treatment. Follow-up of each cohort member continued until the date of the diagnosis of HF from the Hospital Discharge Register, Causes of Death Register or from the National Social Insurance Institution's Drug Reimbursement Register, or death resulting from causes other than HF, or 31 December 2007. The overall positive predictive value of HF diagnosis in this FINRISK study was $85.9 \%$ (negative predictive value $97.9 \%$ ). ${ }^{15}$

\section{Statistical analyses}

Serum GGT levels were classified into five groups using the 25th, 50th, 75th and 90th percentiles as cut-points (quartiles with the top quartile split). The cut-points were 17.1, 25.7, 40.1 and $68.0 \mathrm{U} / 1$ among men, and 11.0, 15.1, 22.1 and $35.0 \mathrm{U} / \mathrm{l}$ among women for the categories of GGT used, respectively. Differences in risk factors based on different levels of serum GGT were tested using General Linear Models after adjustment for age and study year. Cox proportional hazards regression models were used to analyse the association of serum GGT level with the risk of incident HF. All proportionality assumptions were appropriate. The analyses were first carried out adjusting for age, study area and study year at baseline, then for smoking, education, alcohol consumption and physical activity at baseline, and further for BMI, history of valvular heart disease, systolic blood pressure and total cholesterol at baseline, myocardial infarction and diabetes at baseline and during follow-up. Diabetes and myocardial infarction at baseline and during follow-up were used as time-dependent covariates in Cox models. To avoid a potential bias due to severe disease at baseline, additional analyses were carried out excluding the subjects who died during the first 2 years of follow-up $(n=289)$, and subjects who were diagnosed with liver cirrhosis at baseline and during follow-up $(n=223)$. Statistical analyses were performed with PASW for Windows, V.19.0 (IBM SPSS Inc, Chicago, Illinois, USA).

\section{RESULTS}

During a mean follow-up period of 14.5 years, 636 men and 445 women developed HF. General characteristics of the study population by different levels of GGT at baseline are presented in table 1. High levels of GGT were strongly associated with alcohol consumption. The age-adjusted, study area-adjusted and study year-adjusted partial correlations were 0.22 in men $(\mathrm{p}<0.001)$, and 0.11 in women $(\mathrm{p}<0.001)$ for GGT and alcohol consumption.

The age-adjusted, study area-adjusted and study year-adjusted HRs of HF at five GGT groups (using the 25th, 50th, 75th and 90th percentiles) were $1.00,1.46,1.60,2.09$ and 3.13 $\left(\mathrm{P}_{\text {trend }}<0.001\right)$ among men, and $1.00,1.12,1.64,1.92$ and $2.79\left(\mathrm{P}_{\text {trend }}<0.001\right)$ among women (table 2$)$. Considering the strong associations between serum GGT and many CVDs, we presented a model further adjusting for smoking, education, alcohol consumption and physical activity at baseline, and not adjusting any clinical variable in order to avoid over-adjustment. Like the previous model, serum GGT predicted incident HF even in the normal range. Further adjustment for other clinical risk factors (history of valvular heart disease, BMI, systolic blood pressure, total cholesterol at baseline, myocardial infarction and diabetes at baseline and during follow-up) attenuated this relationship; however, the highest category of GGT was still associated with a higher risk of HF in both men (HR 1.79; 95\% CI 1.31 to 2.43 ) and women (HR 1.76 ; 95\% CI 1.25 to 2.48). When men and women were combined, the sex-adjusted and multivariable-adjusted HRs of HF across categories of GGT were $1.00,1.16$ (95\% CI 0.97 to 1.38$), 1.20$ (95\% CI 1.00 to 1.45), 1.29 (95\% CI 1.04 to 1.60$)$, and 1.82 (95\% CI 1.45 to 2.29) $\left(\mathrm{P}_{\text {trend }}<0.001\right)$, which suggested that the risk of HF significantly increased from the 50th to the 90th percentiles. Exclusion of the participants who died during the first 2 years of follow-up ( $n=289)$, and subjects who were diagnosed with liver cirrhosis at baseline and during follow-up $(n=223)$, did not appreciably change the results above (data not shown).

When stratified by age at 60 years, the association between serum GGT and HF risk was only observed among subjects $<60$ years; the multivariable-adjusted HRs of HF across categories of GGT were 1.00, 1.36 (95\% CI 1.09 to 1.71$), 1.30$ (95\% CI 1.02 to 1.66$), 1.41$ (95\% CI 1.07 to 1.86$)$ and 1.90 (95\% CI 1.42 to 2.55$)\left(\mathrm{P}_{\text {trend }}=0.001\right) \quad($ table 3$)$. Stratification by smoking status, alcohol consumption and BMI gave similar results to the pooled multivariable-adjusted HRs in table 2. The interaction between age and GGT was significant $(p=0.014)$, while no significant interactions between GGT and other stratification variables were found. Of note, after stratification by alcohol consumption, the positive association between the highest category of serum GGT and HF risk was observed in both non-drinkers and drinkers. 
Table 1 General characteristics of study subjects at baseline*

\begin{tabular}{|c|c|c|c|c|c|c|}
\hline & \multicolumn{5}{|c|}{ Baseline GGT level } & \multirow[b]{2}{*}{$p$ for trend } \\
\hline & $<25 \%$ & 25 to $<50 \%$ & 50 to $<75 \%$ & 75 to $<90 \%$ & $\geq 90 \%$ & \\
\hline Men $(n=18353)$ & $<17.1 \mathrm{U} / \mathrm{l}$ & 17.1 to $<25.7 \mathrm{U} / \mathrm{l}$ & 25.7 to $<40.1 \mathrm{U} / \mathrm{l}$ & 40.1 to $<68.0 \mathrm{U} / \mathrm{l}$ & $\geq 68.0 \mathrm{U} / \mathrm{l}$ & \\
\hline Subjects (n) & 4659 & 4517 & 4593 & 2739 & 1845 & \\
\hline Age at baseline (years) & 44.3 & 46.3 & 48.1 & 47.8 & 48.8 & $<0.001$ \\
\hline Body mass index $\left(\mathrm{kg} / \mathrm{m}^{2}\right)$ & 25.0 & 26.2 & 27.4 & 28.4 & 29.0 & $<0.001$ \\
\hline Diastolic blood pressure $(\mathrm{mm} \mathrm{Hg})$ & 81 & 83 & 86 & 88 & 90 & $<0.001$ \\
\hline Systolic blood pressure $(\mathrm{mm} \mathrm{Hg})$ & 138 & 139 & 142 & 143 & 147 & $<0.001$ \\
\hline Serum cholesterol (mmol/l) & 5.53 & 5.72 & 5.93 & 6.14 & 6.26 & $<0.001$ \\
\hline Education (years) & 10.6 & 10.5 & 10.5 & 10.4 & 10.5 & 0.538 \\
\hline Alcohol drinker (\%) & 55.5 & 62.5 & 67.9 & 73.6 & 76.9 & $<0.001$ \\
\hline Current smoker (\%) & 28.4 & 35.0 & 38.0 & 42.0 & 45.7 & $<0.001$ \\
\hline Low physical activity (\%) & 6.6 & 6.9 & 9.2 & 11.5 & 13.5 & $<0.001$ \\
\hline History of myocardial infarction (\%) & 3.9 & 3.9 & 3.4 & 4.4 & 5.2 & 0.011 \\
\hline History of valvular heart disease (\%) & 0.2 & 0.2 & 0.2 & 0.2 & 0.3 & 0.755 \\
\hline History of diabetes (\%) & 2.4 & 2.3 & 3.0 & 2.9 & 4.6 & $<0.001$ \\
\hline Women $(n=19726)$ & $<11.0 \mathrm{U} / \mathrm{l}$ & 11.0 to $<15.1 \mathrm{U} / \mathrm{l}$ & 15.1 to $<22.1 \mathrm{U} / \mathrm{l}$ & 22.1 to $<35.0 \mathrm{U} / \mathrm{l}$ & $\geq 35.0 \mathrm{U} / \mathrm{l}$ & \\
\hline Subjects (n) & 4598 & 5572 & 4756 & 2793 & 2007 & \\
\hline Age at baseline (years) & 41.7 & 44.3 & 47.2 & 49.9 & 51.7 & $<0.001$ \\
\hline Body mass index $\left(\mathrm{kg} / \mathrm{m}^{2}\right)$ & 24.7 & 25.4 & 26.4 & 27.6 & 28.6 & $<0.001$ \\
\hline Diastolic blood pressure $(\mathrm{mm} \mathrm{Hg})$ & 78 & 79 & 81 & 82 & 83 & $<0.001$ \\
\hline Systolic blood pressure $(\mathrm{mm} \mathrm{Hg})$ & 132 & 134 & 136 & 138 & 140 & $<0.001$ \\
\hline Serum cholesterol (mmol/l) & 5.60 & 5.68 & 5.72 & 5.81 & 5.91 & $<0.001$ \\
\hline Education (years) & 11.2 & 11.2 & 11.1 & 11.0 & 10.7 & $<0.001$ \\
\hline Alcohol drinker (\%) & 39.5 & 44.6 & 47.9 & 50.2 & 52.5 & $<0.001$ \\
\hline Current smoker (\%) & 13.5 & 18.9 & 23.3 & 25.6 & 29.3 & $<0.001$ \\
\hline Low physical activity (\%) & 7.1 & 7.9 & 9.7 & 11.0 & 14.7 & $<0.001$ \\
\hline History of myocardial infarction (\%) & 0.8 & 0.9 & 0.9 & 1.6 & 1.6 & 0.001 \\
\hline History of valvular heart disease (\%) & 0.2 & 0.1 & 0.2 & 0.0 & 0.3 & 0.005 \\
\hline History of diabetes (\%) & 1.4 & 1.7 & 1.8 & 2.8 & 5.0 & $<0.001$ \\
\hline
\end{tabular}

*Baseline characteristics represent mean or percentage; adjusted for age, study area and study year.

Table 2 HRs of heart failure according to different levels of serum GGT

\begin{tabular}{|c|c|c|c|c|c|c|}
\hline & \multicolumn{5}{|c|}{ Baseline serum GGT level } & \multirow[b]{2}{*}{$\mathrm{p}$ for trenc } \\
\hline & $<25 \%$ & 25 to $<50 \%$ & 50 to $<75 \%$ & 75 to $<90 \%$ & $\geq 90 \%$ & \\
\hline \multicolumn{7}{|l|}{ Men } \\
\hline Incidence case $(\mathrm{n})$ & 149 & 153 & 151 & 100 & 83 & \\
\hline Person-years & 83542 & 63716 & 58828 & 34195 & 20886 & \\
\hline Age, area and study years adjusted $\mathrm{HR}(95 \% \mathrm{Cl})$ & 1.00 & 1.46 (1.16 to 1.84$)$ & 1.60 (1.27 to 2.02$)$ & 2.09 (1.61 to 2.71$)$ & $3.13(2.37$ to 4.14$)$ & $<0.001$ \\
\hline Multivariable adjustment $\mathrm{HR}(95 \% \mathrm{Cl})^{*}$ & 1.00 & 1.41 (1.12 to 1.77$)$ & 1.51 (1.19 to 1.91$)$ & $1.92(1.47$ to 2.51$)$ & 2.70 (2.02 to 3.62$)$ & $<0.001$ \\
\hline Multivariable adjustment $\mathrm{HR}(95 \% \mathrm{Cl}) \dagger$ & 1.00 & 1.25 (1.00 to 1.58$)$ & $1.18(0.92$ to 1.50$)$ & $1.32(1.00$ to 1.74$)$ & 1.79 (1.31 to 2.43$)$ & 0.006 \\
\hline \multicolumn{7}{|l|}{ Women } \\
\hline Incidence case $(\mathrm{n})$ & 98 & 115 & 102 & 63 & 67 & \\
\hline Person-years & 91860 & 86478 & 58944 & 32512 & 22844 & \\
\hline Age, area and study years adjusted HR $(95 \% \mathrm{Cl})$ & 1.00 & 1.12 (0.85 to 1.46$)$ & 1.64 (1.24 to 2.18$)$ & 1.92 (1.39 to 2.66$)$ & 2.79 (2.02 to 3.84$)$ & $<0.001$ \\
\hline Multivariable adjustment $\mathrm{HR}(95 \% \mathrm{Cl})^{*}$ & 1.00 & 1.13 (0.86 to 1.48$)$ & 1.59 (1.20 to 2.11$)$ & $1.83(1.32$ to 2.54$)$ & 2.60 (1.88 to 3.59$)$ & $<0.001$ \\
\hline Multivariable adjustment $\mathrm{HR}(95 \% \mathrm{Cl}) \dagger$ & 1.00 & $0.98(0.75$ to 1.29$)$ & 1.19 (0.89 to 1.59$)$ & $1.21(0.86$ to 1.70$)$ & $1.76(1.25$ to 2.48$)$ & 0.006 \\
\hline \multicolumn{7}{|l|}{ Men and women combined $\neq$} \\
\hline Incidence case $(\mathrm{n})$ & 247 & 268 & 253 & 163 & 150 & \\
\hline Person-years & 175403 & 150193 & 117772 & 66707 & 43729 & \\
\hline Age, area and study years adjusted HR (95\% Cl) & 1.00 & $1.33(1.12$ to 1.59$)$ & $1.64(1.37$ to 1.97$)$ & 2.05 (1.67 to 2.50$)$ & 3.08 (2.49 to 3.80$)$ & $<0.001$ \\
\hline Multivariable adjustment $\mathrm{HR}(95 \% \mathrm{Cl})^{*}$ & 1.00 & $1.30(1.09$ to 1.55$)$ & 1.57 (1.31 to 1.88$)$ & 1.91 (1.55 to 2.34$)$ & $2.74(2.21$ to 3.40$)$ & $<0.001$ \\
\hline Multivariable adjustment $\mathrm{HR}(95 \% \mathrm{Cl}) \dagger$ & 1.00 & $1.16(0.97$ to 1.38$)$ & $1.20(1.00$ to 1.45$)$ & $1.29(1.04$ to 1.60$)$ & $1.82(1.45$ to 2.29$)$ & $<0.001$ \\
\hline
\end{tabular}


Table 3 HRs of heart failure according to different levels of serum GGT stratified by age, smoking status, alcohol consumption and BMI*

\begin{tabular}{|c|c|c|c|c|c|c|c|}
\hline & \multirow[b]{2}{*}{ Cases (n) } & \multicolumn{5}{|c|}{ Baseline GGT level } & \multirow[b]{2}{*}{$p$ for trend } \\
\hline & & $<25 \%$ & 25 to $<50 \%$ & 50 to $<75 \%$ & 75 to $<90 \%$ & $\geq 90 \%$ & \\
\hline \multicolumn{8}{|c|}{ Age at baseline (years) } \\
\hline $25-60$ & 655 & 1.00 & $1.36(1.09-1.71)$ & $1.30(1.02-1.66)$ & $1.41(1.07-1.86)$ & $1.90(1.42-2.55)$ & 0.001 \\
\hline $60-74$ & 426 & 1.00 & $0.93(0.70-1.24)$ & $1.10(0.82-1.48)$ & $1.03(0.73-1.47)$ & $1.43(0.99-2.08)$ & 0.173 \\
\hline \multicolumn{8}{|l|}{ Smoking status } \\
\hline Never or ever & 737 & 1.00 & $1.02(0.83-1.26)$ & $1.14(0.92-1.42)$ & $1.21(0.93-1.57)$ & $1.67(1.26-2.20)$ & 0.004 \\
\hline Current & 344 & 1.00 & $1.48(1.06-2.08)$ & $1.37(0.97-1.95)$ & $1.51(1.02-2.25)$ & $2.29(1.51-3.46)$ & 0.003 \\
\hline \multicolumn{8}{|c|}{ Alcohol consumption } \\
\hline Never drinker & 614 & 1.00 & $1.13(0.89-1.39)$ & $1.13(0.88-1.43)$ & $1.50(1.13-2.00)$ & $1.99(1.47-2.70)$ & $<0.001$ \\
\hline Alcohol drinker & 467 & 1.00 & $1.20(0.89-1.60)$ & $1.32(0.99-1.76)$ & $1.15(0.82-1.60)$ & $1.80(1.28-2.52)$ & 0.010 \\
\hline \multicolumn{8}{|c|}{ Body mass index $\left(\mathrm{kg} / \mathrm{m}^{2}\right)$} \\
\hline$<25$ & 239 & 1.00 & $1.21(0.85-1.72)$ & $1.60(1.10-2.33)$ & $1.99(1.24-3.20)$ & $3.28(2.02-5.33)$ & $<0.001$ \\
\hline $25-29.9$ & 448 & 1.00 & $1.13(0.88-1.47)$ & $1.06(0.80-1.41)$ & $1.15(0.83-1.60)$ & $1.58(1.10-2.26)$ & 0.161 \\
\hline$\geq 30$ & 394 & 1.00 & $1.13(0.80-1.61)$ & $1.15(0.81-1.63)$ & $1.24(0.85-1.82)$ & $1.66(1.12-2.45)$ & 0.094 \\
\hline
\end{tabular}

\section{DISCUSSION}

The present study suggested a positive association between serum GGT and the risk of HF. This association was more pronounced among subjects aged $<60$ years, which is in line with the finding of our previous study ${ }^{2}$ which evaluated the association between serum GGT and incident CHD with the FINRISK dataset. A similar trend was also observed in two other studies $^{67}$ on the association of GGT with CVD mortality. Lee et $a l^{7}$ proposed that the observed age difference might be explained by the difference in the ability of maintaining homeostasis $^{16}$ and clearing xenobiotics ${ }^{17}$ in people of different ages. Xenobiotics, which could be understood as substances foreign to an entire biological system, were directly associated with CVD. ${ }^{18}{ }^{19}$ Serum GGT has been considered as an exposure marker of xenobiotics. ${ }^{20}$ However, serum GGT may not reflect the extent of exposure to xenobiotics in older people as in younger people because older people have a reduced hepatic ability to clear xenobiotics, and serum GGT may increase proportional to the amount of glutathione conjugates, but not the amount of xenobiotics. ${ }^{7}$ Therefore, the prognostic role of serum GGT in older subjects was not as strong as in younger subjects.

Besides the association of serum GGT with incident CVD or CVD mortality that have already been observed, ${ }^{3-8} 2930$ the other clue that led us to investigate the association between serum GGT and incident HF was that serum GGT has been found to be a risk marker for hypertension, ${ }^{21} \mathrm{CHD},{ }^{2}$ insulin resistance, ${ }^{22}$ type 2 diabetes, ${ }^{21}$ dyslipidemia ${ }^{23}$ and inflammation, ${ }^{24}$ all of which are known risk factors of HF. In our previous study, ${ }^{2}$ it was shown that a high level of serum GGT was associated with an increased risk of CHD. The current findings on the relationship between serum GGT and the risk of HF are similar to the previous findings about the association between serum GGT and the risk of CHD. In the present study, the positive association between serum GGT and the risk of HF remained after adjusting for major HF risk factors.

In the present study, alcohol consumption was considered as a confounding factor in the multivariable model and the positive association between serum GGT, and the risk of HF was found in both alcohol and non-alcohol drinkers, which suggests that there are other mechanisms linking serum GGT to the risk of HF besides liver pathology. However, given the temporal lag between the assessment of serum GGT at baseline and the measurement of the outcome, it is not possible to determine the pathways by which GGT resulted in higher risk of HF. Although the precise underlying mechanisms remain unclear, oxidative stress, ${ }^{25} 26$ exposure to environmental pollutants ${ }^{18}$ and non-alcoholic fatty liver disease (NAFLD), ${ }^{27}$ all of which are considered to be involved in the pathogenesis of CVD, were proposed to be partly responsible for the observed association. Serum GGT may predict CVD as a marker of oxidative stress related to glutathione (GSH), ${ }^{28}$ exposure to environmental pollutants which need to be conjugated to $\mathrm{GSH}^{5}$ and NAFLD. ${ }^{29}$ Also, previous results of our group have shown that the correlation between GGT and $\mathrm{N}$-terminal prohormone of brain natriuretic peptide was weak $(r=-0.09)$ in the FINRISK 1997 cohort, which suggests that the elevation of GGT is not secondary to early subclinical HF. ${ }^{30}$

There are several strengths and limitations in our study. First, a major strength of the study is the large number of both men and women from a homogeneous population who participated in the study. Second, the mean follow-up period was sufficiently long to ascertain a large number of HF endpoint events. Finally, we also carried out additional analyses excluding the subjects who died during the first 2 years of follow-up, and subjects who were diagnosed with liver cirrhosis at baseline and during follow-up to avoid a potential bias due to a severe disease at baseline, and confounding from liver disease at baseline and during follow-up. A limitation of our study is that GGT was recorded only once at baseline. Therefore, we are not able to test the reproducibility and validity of GGT reflecting liver dysfunction. In addition, we cannot completely either exclude the effects of residual confounding due to measurement error in the assessment of confounding factors, or some unmeasured factors such as other causes for HF.

In conclusion, our study indicated a direct association between serum GGT and the risk of HF in men and women in Finland, especially in those aged $<60$ years, and this association was independent of self-reported alcohol intake. Although GGT is often measured as a marker of liver health, this study provides evidence that it may also be useful in the identification of patients at elevated risk of CVD and HF. Future studies are required to determine the clinical utility of serum GGT in monitoring subjects at risk of developing HF. 
Contributors GH had full access to all the data in the study, and takes responsibility for the integrity of the data and the accuracy of the data analysis. Study concept and design: YW, GH. Analysis and interpretation of data: YW, GH, JT, PJ, VS, RA, MM, PTK, BL. Drafting of the manuscript: YW, GH. Critical revision of the manuscript for important intellectual content: YW, GH, JT, PJ, VS, RA, MM, PTK, BL. Study supervision: GH.

Funding This work was supported by grants from the Finnish Academy (108297 and 118065), and Special Research Funds of the Social Welfare and Health Board, City of Oulu.

Competing interests None.

Provenance and peer review Not commissioned; internally peer reviewed.

\section{REFERENCES}

1 Lloyd-Jones DAdams RJ, Brown TM, et al. Heart disease and stroke statistics-2010 update: a report from the American heart association. Circulation 2010;121:e46-e215.

2 Lee $\mathrm{DH}$, Silventoinen $\mathrm{K}, \mathrm{Hu} \mathrm{G}$, et al. Serum gamma-glutamyltransferase predicts non-fatal myocardial infarction and fatal coronary heart disease among 28,838 middle-aged men and women. Eur Heart J 2006;27:2170-6.

3 Fraser A, Harris R, Sattar N, et al. Gamma-glutamyltransferase is associated with incident vascular events independently of alcohol intake analysis of the British women's heart and health study and meta-analysis. Arterioscler Thromb Vasc Biol 2007;27:2729-35.

4 Dhingra R, Gona P, Wang TJ, et al. Serum gamma-glutamyltransferase and risk of heart failure in the community. Arterioscler Thromb Vasc Biol 2010:30:1855-60.

5 Strasak AM, Kelleher CC, Klenk J, et al. Longitudinal change in serum gamma-glutamyltransferase and cardiovascular disease mortality-A prospective population-based study in 76113 Austrian adults. Arterioscler Thromb Vasc Biol 2008:28:1857-65.

6 Ruttmann E, Brant LJ, Concin H, et al. Gamma-glutamyltransferase as a risk factor for cardiovascular disease mortality: an epidemiological investigation in a cohort of 163944 Austrian adults. Circulation 2005;112:2130-7.

7 Lee DH, Buijsse B, Steffen L, et al. Association between serum gammaglutamyltransferase and cardiovascular mortality varies by age: the Minnesota Heart Survey. Eur J Cardiovasc Prev Rehabil 2009;16:16-20.

8 Emdin M, Passino C, Pompella A, et al. Gamma-glutamyltransferase as a cardiovascular risk factor. Eur Heart J 2006;27:2145-6.

9 Paolicchi A, Emdin M, Ghliozeni E, et al. Images in cardiovascular medicine. Human atherosclerotic plaques contain gamma-glutamyl transpeptidase enzyme activity. Circulation 2004;109:1440.

10 Vartiainen $\mathrm{E}$, Laatikainen $\mathrm{T}$, Peltonen $\mathrm{M}$, et al. Thirty-five-year trends in cardiovascular risk factors in Finland. Int J Epidemiol 2010:39:504-18.

11 Pajak A, Kuulasmaa K, Tuomilehto J, et al. Geographical variation in the major risk factors of coronary heart disease in men and women aged 35-64 years. The WHO MONICA Project. World Health Stat Q 1988;41:115-40.

12 Hu G, Jousilahti P, Antikainen R, et al. Joint effects of physical activity, body mass index, waist circumference, and waist-to-hip ratio on the risk of heart failure. Circulation 2010;121:237-44.

13 Wang Y, Tuomilehto J, Jousilahti $\mathrm{P}$, et al. Occupational, commuting, and leisure-time physical activity in relation to heart failure among Finnish men and women. J Am Coll Cardiol 2010:56:1140-8.
14 Hu G, Qiao Q, Silventoinen K, et al. Occupational, commuting, and leisure-time physical activity in relation to risk for type 2 diabetes in middle-aged Finnish men and women. Diabetologia 2003:46:322-9.

15 Mahonen $M$, Jula A, Harald $K$, et al. The validity of heart failure diagnoses obtained from administrative registers. Eur, J Prev Cardiolog 2012. http://cpr.sagepub.com/ content/early/2012/02/06/2047487312438979.abstract (accessed November 5 2012)

16 Martin I, Grotewiel MS. Oxidative damage and age-related functional declines. Mech Ageing Dev 2006;127:411-23.

17 Chrysohoou C, Castorini C, Aggelopoulos P, et al. Moderate coffee consumption lowers the likelihood of developing left ventricular systolic dysfunction after an acute coronary event in normotensive, but not in hypertensive patients. J Am College Cardiol 2009;53:A221-A.

$18 \mathrm{Ha} \mathrm{MH}$, Lee $\mathrm{DH}$, Jacobs DR. Association between serum concentrations of persistent organic pollutants and self-reported cardiovascular disease prevalence: results from the National Health and Nutrition Examination Survey, 1999-2002. Environ Health Perspect 2007;115:1204-9.

19 Lee DH, Lee IK, Porta M, et al. Relationship between serum concentrations of persistent organic pollutants and the prevalence of metabolic syndrome among non-diabetic adults: results from the National Health and Nutrition Examination Survey 1999-2002. Diabetologia 2007;50:1841-51.

20 Lee $\mathrm{DH}$, Jacobs DR. Is serum gamma-glutamyltransferase an exposure marker of xenobiotics? Empirical evidence with polycylic aromatic hydrocarbon. Clin Chem Lab Med 2009;47:860-2.

21 Lee $\mathrm{DH}$, Jacobs DR, Gross $\mathrm{M}$, et al. gamma-glutamyltransferase is a predictor of incident diabetes and hypertension: the coronary artery risk development in young adults (CARDIA) study. Clin Chem 2003;49:1358-66.

22 Ishizaka N, Ishizaka Y, Toda El, et al. Association between GammaGlutamyltransferase levels and insulin resistance according to alcohol consumption and number of cigarettes smoked. J Atheroscler Thromb 2010;17:476-85.

23 Ruttmann E, Brant LJ, Concin $\mathrm{H}$, et al. gamma-glutamyltransferase as a risk factor for cardiovascular disease mortality-An epidemiological investigation in a cohort of 163,944 Austrian adults. Circulation 2005;112:2130-7.

24 Yamada J, Tomiyama H, Yambe $M$, et al. Elevated serum levels of alanine aminotransferase and gamma glutamyltransferase are markers of inflammation and oxidative stress independent of the metabolic syndrome. Atherosclerosis 2006;189:198-205

25 Dhalla NS, Temsah RM, Netticadan T. Role of oxidative stress in cardiovascular diseases. J Hypertens 2000;18:655-73.

26 Arab L. Epidemiologic evidence on coffee and cancer. Nutr Cancer 2010;62:271-83.

27 Targher G, Arcaro G. Non-alcoholic fatty liver disease and increased risk of cardiovascular disease. Atherosclerosis 2007;191:235-40.

28 Lim JS, Yang JH, Chun BY, et al. Is serum gamma-glutamyltransferase inversely associated with serum antioxidants as a marker of oxidative stress?. Free Radic Biol Med 2004;37:1018-23.

29 Kastorini CM, Chrysohoou C, Panagiotakos D, et al. Moderate coffee consumption lowers the likelihood of developing left ventricular systolic dysfunction in post-acute coronary syndrome normotensive patients. J Med Food 2009;12:29-36.

30 Salomaa V, Havulinna A, Saarela 0, et al. Thirty-one novel biomarkers as predictors for clinically incident diabetes. PLoS One 2010;5:e10100. 